\title{
Ambiente e Salute News n.12 novembre-dicembre 2021
}

\author{
A cura di Giacomo Toffol e Vincenza Briscioli \\ Gruppo ACP Pediatri per Un Mondo Possibile
}

\begin{abstract}
Questo numero va in stampa a breve distanza dall'inserimento nella Costituzione Italiana di una legge a Tutela dell'Ambiente, (8 febbraio 2022) che modifica due articoli: l'articolo 9 in cui viene inserito il testo "la Repubblica Italiana ...Tutela l'ambiente, la biodiversità e gli ecosistemi, anche nell'interesse delle future generazioni." e l'articolo 41 "L'iniziativa economica privata .... non può svolgersi in contrasto con l'utilità sociale o in modo da recare danno alla sicurezza, alla libertà, alla dignità umana, alla salute e all'ambiente." Si tratta di un primo passo nell'ottica promossa dall'Alleanza Globale per i Diritti della Natura: inserire i diritti della Natura o della Terra nell'ambito delle Costituzioni attribuisce ad esse un valore giuridico (https://www.garn.org/). È certo di buon auspicio aver messo per iscritto l'interesse delle future generazioni e la tutela della biodiversità e proprio per questo riteniamo utile sottolineare come sia ora necessario passare ad atti concreti, di controllo per esempio di situazioni inquinanti del suolo, dell'aria, dell'acqua che necessitano di essere risolte e che non possono più tollerare ritardi. Anche in questo numero, in cui vengono riassunti sinteticamente i principali articoli pubblicati nelle riviste monitorate, molti sono i dati raccolti che dimostrano come la contaminazione ambientale danneggi la salute dell'uomo e come il diritto dell'uomo alla salute combaci con il dovere della tutela dell'ambiente ed il diritto della Terra ad essere tutelata. Questo numero si basa sul controllo sistematico delle pubblicazioni di Novembre e Dicembre 2021.
\end{abstract}

Environment and health

This issue goes to press shortly after the inclusion in the Italian Constitution of a law on Environmental Protection, (8th February 2022) amending two articles: Article 9 in which is added the text: "the Italian Republic ... protects environment, biodiversity and ecosystems, also in the interest of future generations", and Article 41 in which is added the text: "Private economic initiative... may not be carried out in a manner contrary to social utility or which adversely affects security, freedom, human dignity, health and the environment." This is a first step towards the perspective promoted by the Global Alliance for the Rights of Nature: including the rights of Nature or of the Earth in the Constitutions gives them a legal value (https://www.garn.org/). It certainly bodes well to have written down the interests of future generations and the protection of biodiversity. Precisely for this reason we believe that It is important to stress the need to move on to concrete actions, such the necessity to control soil, air and water pollution. All this needs to be resolved now, and further delays are not tolerable. In this issue, which summarizes the main articles published in the monitored journals, a great deal of data has been collected showing that environmental contamination harms human health, and that human health rights are in line with both the duty to protect environment and the right of the Earth to be protected. This issue is based on the systematic monitoring of the November and December 2021 publications.

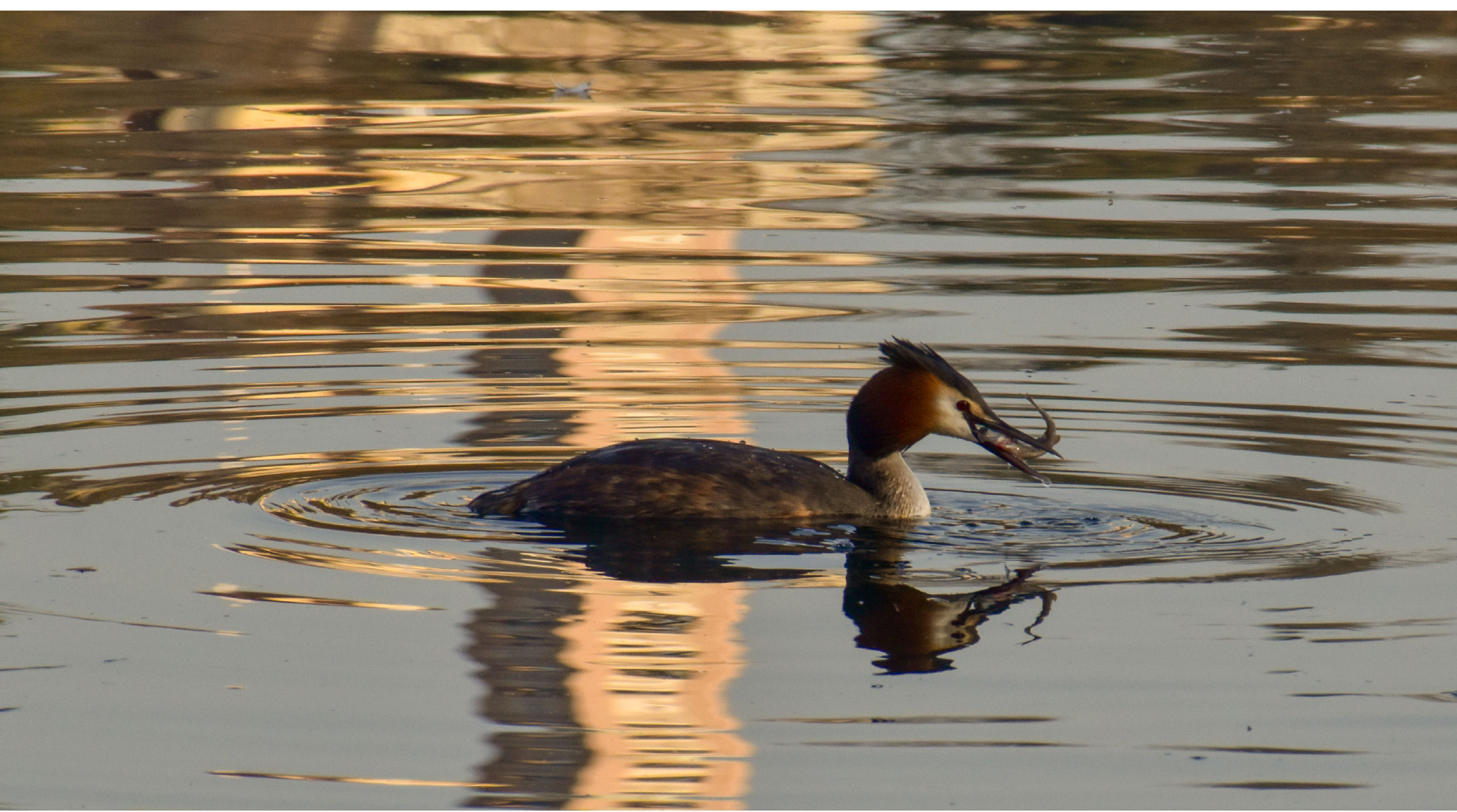

La fotografia di questo numero è stata concessa dagli organizzatori del concorso fotografico "Noi siamo la natura" 


\section{Ambiente e Salute News}

\section{Indice}

\section{:: Inquinamento atmosferico}

1. Bicicletta in sostituzione dell'auto ed effetti sulla salute pubblica: possibile riduzione della mortalità prematura

2. Qualità dell'aria prima e dopo l'inizio della pandemia COVID-19 in 87 città industriali ed inquinate

3. Inquinamento atmosferico da traffico e rischio di nascite pretermine in California

4. Effetti dell'esposizione al particolato fine indoor sulla dermatite atopica dei bambini

5. Esposizione al particolato durante la gravidanza e sviluppo multidimensionale in bambini di età scolare: uno studio trasversale italiano

6. Esposizione pre e postnatale al black carbon e al particolato atmosferico e flusso sanguigno nel microcircolo cutaneo

7. Esposizione a particolato ultrafine, funzione polmonare e sintomi respiratori in adolescenti asmatici e sani

\section{:: Inquinamento da sostanze chimiche non atmosferiche}

1. Consensus italiana sulla gestione clinica e terapeutica della 'Sensibilità chimica multipla' (MCS, Multiple Chemical Sensitivity)

2. Esposizione materna agli ftalati e valori pressori durante e dopo la gravidanza nello studio PROGRESS

3. Esposizione prenatale a miscele chimiche non persistenti e crescita fetale: uno studio di popolazione

4. Esposizione ai rifiuti elettronici e salute: una revisione sistematica

5. Aumento di prevalenza di dermatite atopica in bambini di età compresa tra 0-3 anni altamente esposti ai parabeni

6. Agricoltura di città, benefici e rischi

7. Assunzione di farmaci nitrosabili, apporto di nitrato con l'acqua potabile e rischio di natimortalità in una coorte di donne danesi

8. Esposizione agli ftalati e tratti autistici nell'infanzia

9. I composti per e polifluoralchilici (PFAS) alterano la composizione lipidica del latte materno

10. Esposizione prenatale e postnatale ai PFAS, fattori cardiometabolici e stato di infiammazione nei bambini di 6 coorti europee

11. Glifosato e acido aminometilfosfonico (AMPA) nelle urine di bambini ed adolescenti in Germania: uno studio di biomonitoraggio

12. Esposizione perinatale a potenziali interferenti endocrini e rischio di criptorchidismo in Norvegia

13. Associazione tra una miscela di fenoli e ftalati e comportamento del bambino

\section{:: Psicologia ambientale}

1. Effetti a breve e lungo termine degli incentivi economici sulla volontà di utilizzare i mezzi pubblici per recarsi al lavoro

2. Comprendere le intenzioni dei genitori di consentire ai loro figli di andare a scuola a piedi o in bicicletta: il questionario PILCAST

3. I bambini inconsapevoli consumatori di carne: una opportunità per affrontare il cambiamento climatico

4. Le reazione agli avvisi riguardanti il cambiamento climatico nella popolazione

5. La considerazione sulle conseguenze future e la distanza temporale contribuiscono al divario ideologico nello scetticismo sul cambiamento climatico

6. Vedere o essere: il contatto con la natura durante le chiusure dovute alla pandemia da Covid-19

\section{:: Miscellanea}

7. I sogni della Terra: reinventare ARPA-H per la salute di persone, luoghi e pianeta

- Articoli in evidenza

\section{Riviste monitorate}

American Journal of Public Health

American Journal of Respiratory and Critical Care medicine

American Journal of Epidemiology

Archives of Diseases in Childhood

Brain \& Development

British Medical Journal

Child: Care, Health and Development

Environmental and Health

Environmental Health Perspectives

Environmental International

Environmental Pollution

Environmental Research

Environmental Sciences Europe

European Journal of Epidemiology

International Journal of Environmenta Research and Public

Health

International Journal of Epidemiology

JAMA (Journal of American Medical Association)

JAMA Pediatrics

Journal of Environmental Psychology

Journal of Epidemiology and Community Health

Journal of Pediatrics

The Lancet

NeuroToxicology

Neurotoxicology and Teratology

New England Journal of Medicine

Pediatrics

Revisione delle riviste e testi a cura di:

Angela Biolchini, Luisa Bonsembiante, Vincenza Briscioli, Laura Brusadin, Sabrina Bulgarelli, Elena Caneva, Ilaria Mariotti, Federico Marolla, Aurelio Nova, Angela Pasinato, Giuseppe Primavera, Laura Reali, Annamaria Sapuppo, Laura Todesco, Giacomo Toffol, Elena Uga, Anna Valori, Luisella Zanino.

\section{Pediatri per Un Mondo Possibile}

Gruppo di studio sulle patologie correlate all'inquinamento ambientale dell'Associazione Culturale Pediatri (ACP)

mail: pump@acp.it 


\section{Inquinamento atmosferico}

\section{Bicicletta in sostituzione dell'auto ed effetti sulla salute} pubblica: possibile riduzione della mortalità prematura

La bicicletta gioca un ruolo importante nella mobilità urbana ed è stata proposta come strumento per promuovere la salute pubblica. Lo studio ha analizzato gli effetti dell'utilizzo della bicicletta (in sostituzione dell'auto) sulla salute pubblica misurando le possibili variazioni sulla mortalità prematura in popolazioni urbane in 17 stati. L'effetto è stato valutato considerando la variazione del numero di morti premature (aumento o diminuzione) correlate a incidenti stradali, inquinamento ambientale e attività fisica. Lo studio ha coinvolto una popolazione con età compresa tra i 20 e i 64 anni. Ė emerso che l'utilizzo della bicicletta al posto dell'auto (assumendo che il 100\% degli spostamenti in auto siano sostituiti con la bicicletta) potrebbe prevenire 205.424 morti premature all'anno nella popolazione urbana (20-64 anni) di 17 stati entro il 2050. Se solo l'8\% degli spostamenti in auto venisse sostituito dalla bicicletta, potrebbero essere evitate 18.589 morti premature all'anno nella stessa popolazione entro il 2050. In tutti gli scenari e in tutti i paesi coinvolti i benefici sulla mortalità correlati all'utilizzo della bicicletta superano i rischi. Incentivare l'utilizzo della bicicletta al posto dell' auto potrebbe dunque avere un importante beneficio sulla mortalità delle popolazioni urbane e deve essere considerato uno strumento per migliorare la salute della popolazione.

${ }^{\circ}$ Egiguren, J., Nieuwenhuijsen, M. J., \& Rojas-Rueda, D. (2021). Premature mortality of 2050 high bike use scenarios in 17 countries. Environmental health perspectives, 129(12), 127002

\section{Qualità dell'aria prima e dopo l'inizio della pandemia CO- VID-19 in 87 città industriali ed inquinate}

Lo studio ha analizzato l'impatto della pandemia da COVID-19 sui cambiamenti degli indici di qualità dell'aria (AQI) in diverse capitali densamente abitate prima e dopo il 2020. Lo studio ha utilizzato gli AQI ottenuti da database disponibili gratuitamente come il "World Air Quality Index (WAQI)". È emerso che le variazioni di $\mathrm{PM}_{2.5}, \mathrm{PM}_{10}$ e $\mathrm{NO}_{2}$ erano significativamente più alte prima e dopo il 2020 contemporaneamente alle restrizioni dovute al COVID-19 in diverse città del mondo. Le diminuzioni di $\mathrm{PM}_{2.5}, \mathrm{PM}_{10}$ e $\mathrm{NO}_{2}$ nel 2020 sono state del $-7.36 \%,-17.42 \%$ e $-20.54 \%$ rispetto al 2019. Tuttavia, nel 2021, si è assistito a un incremento degli stessi inquinanti $(+4.25 \%,+9.08 \%,+7.48 \%)$. In generale, inoltre, le temperature e l'umidità relativa sono risultate essere inversamente correlate a $\mathrm{PM}_{2.5}, \mathrm{PM}_{10}$ e $\mathrm{NO}_{2}$. Anche dopo l'aggiustamento considerando altri fattori meteo l'umidità relativa è risultata essere inversamente associata ai valori dei tre inquinanti. I risultati suggeriscono che durante il 2020 si è assistito a un miglioramento dei livelli di inquinanti ambientali. Tale effetto, tuttavia, non si è verificato nel 2021, probabilmente in seguito alla riduzione delle restrizioni seguite in diversi stati. Nonostante gli effetti della pandemia abbiano portato a miglioramenti della qualità dell'aria solo temporanei, questa conseguenza può essere importante per pianificare un controllo degli inquinanti ambientali.

- SARMADI, Mohammad, et al. Air quality index variation before and after the onset of COVID-19 pandemic: a comprehensive study on 87 capital, industrial and polluted cities of the world. Environmental Sciences Europe, 2021, 33.1: 1-17

\section{Inquinamento atmosferico da traffico e rischio di nascite pretermine in California}

Questo studio analizza l'impatto che potrebbe avere l'inquinamento atmosferico legato al traffico (TRAP) nell'incrementare il rischio di parto pretermine e l'effetto a distanza sui neonati. I dati dei registri delle nascite sono stati correlati con il TRAP a livello stradale nel periodo 2013-2015 a Oakland e San Jose in California, stimando gli effetti dellesposizione al black carbon, al biossido di azoto e alle particelle ultrafini sulle nascite pretermine singole tenendo conto di variabili confondenti e stratificando per etnia. Su un totale di 8.823 nascite singole, 760 (8.6\%) erano pretermine. Un passaggio dal $10^{\circ}$ al $90^{\circ}$ percentile del livello di esposizione al black carbon è associato a: $6.8 \%$ di rischio più elevato di parto pretermine (IC $95 \% 0.1$ - 13.5) tra le donne di colore; $2.1 \%$ di rischio più alto tra le donne di etnia latina (IC $95 \%$ -1.1 - 5.2). I risultati non sono conclusivi per le donne asiatiche e bianche. Per le latine, c'era inoltre evidenza di un'associazione statisticamente significativa tra gli altri inquinanti ed il rischio di parto pretermine, sebbene le dimensioni dell'effetto si riducessero quando nell'analisi si teneva conto anche degli altri TRAP. In conclusione sembra che l'esposizione a TRAP, in particolare al black carbon, possa aumentare il rischio di parto pretermine per le donne di etnia latina e nera, ma apparentemente non per le donne asiatiche e bianche.

${ }^{\circ}$ RIDDELL, Corinne A., et al. Hyper-localized measures of air pollution and risk of preterm birth in Oakland and San Jose, California. International journal of epidemiology, 2021, 50.6: 1875-1885

\section{Effetti dell'esposizione al particolato fine indoor sulla der- matite atopica dei bambini}

Questo studio mirava a studiare l'effetto a breve termine dell' esposizione al particolato fine $\left(\mathrm{PM}_{2.5}\right)$ indoor sui sintomi della dermatite atopica (AD) nei bambini. Sono stati inclusi 64 bambini con $\mathrm{AD}$ da moderata a grave, di età inferiore a 18 anni, nel periodo da febbraio 2019 a novembre 2020. L'esposizione a $\mathrm{PM}_{2.5}$ indoor dei pazienti ed i loro sintomi di $\mathrm{AD}$ sono stati misurati quotidianamente, stratificando i pazienti per età, sesso, stagione, gravità, presenza di malattie allergiche familiari, sensibilizzazione e condizioni ambientali indoor, comprese la temperatura e l'umidità relativa. La concentrazione media di $\mathrm{PM}_{2.5}$ era $28.7 \pm 24.3 \mu \mathrm{g} / \mathrm{m} 3$, con il valore più alto in inverno $(47.1 \pm 29.6$ $\mu \mathrm{g} / \mathrm{m} 3)$. L'effetto complessivo di $\mathrm{PM}_{2.5}$ sui sintomi di $\mathrm{AD}$ non è risultato statisticamente significativo. Tuttavia, un aumento di 10 $\mu \mathrm{g} / \mathrm{m} 3$ nella concentrazione di $\mathrm{PM}_{2.5}$ in ambienti chiusi comporta un incremento dei punteggi dei sintomi di AD del 16.5\% (IC 95\%: 6.5-27.5) in primavera e del 12.6\% (IC 95\%: 4.3-21.5) in inverno, del 6.7\% (IC 95\%: 2.3-11.3) a temperature interne $<25.5^{\circ}$ C e del 15.0\% (IC 95\%: 3.5-27.7) in assenza di un purificatore d'aria. L'effetto dannoso del $\mathrm{PM}_{25}$ nei ragazzi, nei bambini di età $\geq 6$ anni e nei bambini con sensibilizzazione agli allergeni inalanti erano significativi, mostrando un aumento dei sintomi di AD del 4.9\% (IC 95\%: 1.4-8.6), 12.0\% (IC 95\%: 5.3-19.1) e 7.0\% (IC 95\%: 1.9-12.3) per valori di $10 \mu \mathrm{g} / \mathrm{m} 3$ di $\mathrm{PM}_{2.5}$, rispettivamente. Inoltre, bambini con sensibilizzazione agli allergeni inalanti più 
sintomi gravi (SCORing Atopic Dermatite, SCORAD $\geq 30.7$, valore mediano) hanno mostrato effetti più dannosi da esposizione a $\mathrm{PM}_{2.5}$ (aumento del 15.7\% (IC 95\%: 4.5-28.1) nei punteggi dei sintomi di $\mathrm{AD}$ ogni $10 \mu \mathrm{g} / \mathrm{m} 3$ di incremento di $\mathrm{PM}_{2.5}$. In sintesi, l'esposizione indoor a $\mathrm{PM}_{25}$ ha esacerbato i sintomi di $\mathrm{AD}$ nei bambini in primavera, inverno e a temperature interne $<25.5^{\circ}$ C, specialmente nei bambini con sensibilizzazione agli allergeni inalanti e sintomi gravi. Ridurre al minimo l'esposizione al $\mathrm{PM}_{2.5}$ indoor è pertanto necessario per la corretta gestione della dermatite atopica.

${ }^{\circ}$ KIM, Young-Min, et al. Effects of Exposure to Indoor Fine Particulate Matter on Atopic Dermatitis in Children. International journal of environmental research and public health, 2021, 18.21: 11509

\section{Esposizione al particolato durante la gravidanza e sviluppo multidimensionale in bambini di età scolare: uno studio tra- sversale italiano}

Gli autori hanno indagato il legame tra l'esposizione prenatale al particolato $\left(\mathrm{PM}_{10}\right)$ e lo sviluppo in più domini nei bambini in età scolare. I dati di uno studio trasversale sono stati raccolti in Italia tra il 2013 ed il 2014. 1187 bambini di età tra 5 e gli 8 anni sono stati valutati per i domini cognitivo, comunicativo, socio-emotivo, adattativo e di sviluppo motorio usando uno specifico questionario (Developmental Profile 3). La concentrazione media mensile di $\mathrm{PM}_{10}$ durante l'intero periodo fetale è stata legata al comune di residenza dei bambini. L'incremento del $\mathrm{PM}_{10}$ prenatale era associato ad un decremento del punteggio cognitivo durante il secondo (+13.2 g/m3 $\mathrm{PM}_{10}$ incremento: -0.30 punteggi; $95 \% \mathrm{CI}$ : $-0.12--0.48)$ ed il terzo trimestre gestazionale ( -0.31 punteggi; 95\%CI: -0.11--0.50). Il dominio comunicativo era anch'esso influenzato negativamente dall'incremento del $\mathrm{PM}_{10}$ nel secondo trimestre. Lo sviluppo cognitivo e le abilità di comunicazione dei bambini erano associate negativamente con l'esposizione al $\mathrm{PM}_{10}$ durante il periodo dello sviluppo fetale, confermando che l'esposizione all'inquinamento atmosferico durante la gravidanza può potenzialmente ostacolare lo sviluppo cerebrale.

GIRARDI, Paolo, et al. Association between Exposure to Particulate Matter during Pregnancy and Multidimensional Development in School-Age Children: A Cross-Sectional Study in Italy. International journal of environmental research and public health, 2021, 18.21: 11648

6. Esposizione pre e postnatale al black carbon e al particolato atmosferico e flusso sanguigno nel microcircolo cutaneo In questo studio gli autori hanno valutato l'associazione tra l'esposizione all'inquinamento atmosferico pre e postnatale e l'iperemia cutanea indotta dal calore utilizzata come marker dinamico della microvascolarizzazione e quindi come indice di un buon microcircolo. Sono stati valutati 139 bambini di età compresa tra 4 e 6 anni misurando la perfusione cutanea con sonde Laser Doppler. L'esposizione residenziale ai livelli di black carbon (BC), particolato $\left(\mathrm{PM}_{10}, \mathrm{PM}_{2.5}\right)$ e biossido di azoto $\left(\mathrm{NO}_{2}\right)$ è stata ricavata dall'indirizzo di casa di ogni partecipante utilizzando un modello spazio-temporale ad alta risoluzione per più finestre temporali. Gli autori hanno quindi valutato l'associazione tra iperemia cutanea e esposizione ad inquinamento atmosferico pre e postnatale. I risultati hanno evidenziato come un incremento di esposizione residenziale a $\mathrm{BC}$ durante il terzo trimestre di gravidanza sia associato ad una iperemia cutanea inferiore al $11.5 \%$. Stime di effetti simili sono state evidenziate per l'esposizione a $\mathrm{PM}_{10}, \mathrm{PM}_{2.5} \mathrm{o} \mathrm{NO}_{2}$. Invece l'esposizione postnatale a $\mathrm{BC}, \mathrm{PM}_{10}, \mathrm{PM}_{2.5} \mathrm{O} \mathrm{NO}_{2}$ non è stata associata con variazione dell'iperemia cutanea all'età di 4-6 anni. Gli autori concludono come i risultati ottenuti supportino l'ipotesi che l'esposizione a $\mathrm{BC}$, inquinamento atmosferico da particolato e $\mathrm{NO}_{2}$ anche a basse concentrazioni, durante la vita prenatale, possa essere correlata a conseguenze di lunga durata per il microcircolo. La disfunzione endoteliale gioca un ruolo essenziale nello sviluppo e progressione delle malattie cardiovascolari. Questo studio apre quindi nuove prospettive di indagine sull'iperemia termica come strumento integrativo per valutare la funzione microvascolare e sulla correlazione tra inquinamento atmosferico e microcircolazione nei bambini, che può aggiungere conoscenze alla complicata relazione tra l'esposizione precoce all'inquinamento atmosferico e lo sviluppo di malattie cardiovascolari nelle età più avanzate.

${ }^{\circ}$ WITTERS, Katrien, et al. Dynamics of skin microvascular blood flow in 4-6-year-old children in association with pre-and postnatal black carbon and particulate air pollution exposure. Environment International, 2021, 157: 106799.

\section{Esposizione a particolato ultrafine, funzione polmonare e sintomi respiratori in adolescenti asmatici e sani}

$\mathrm{Ci}$ sono molte evidenze che suggeriscono come il particolato ultrafine (UFPs) sia associato ad alcuni effetti avversi sulla salute, soprattutto su condizioni croniche come l'asma. Lo scopo di questo studio è stato quello di indagare l'associazione tra l'esposizione settimanale al UFPs, la funzione polmonare e i sintomi respiratori in 117 adolescenti asmatici e non asmatici di età compresa tra i 13 e i 17 anni nell'area di Cincinnati. Tra il 2017 e il 2019 i partecipanti hanno raccolto dei campioni di aria nelle loro case, a scuola e nei tragitti percorsi per 3 ore al giorno. Contestualmente, ogni settimana veniva valutata la funzione polmonare e i sintomi respiratori. La mediana di esposizione al UFPs settimanale è risultata essere di 4340 particelle/ $\mathrm{cm} 3(\mathrm{p} / \mathrm{cc})$. È emersa un'associazione negativa tra UFPs e il rapporto tra la percentuale di volume espiratorio forzato e capacità vitale forzata (\%FEV1/FVC). Inoltre è emersa un'associazione tra esposizione al UFPs e sintomi respiratori, soprattutto nei soggetti asmatici. I risultati dunque hanno confermato che il UFPs può essere coinvolto nelle esacerbazioni di una condizione cronica come l'asma.

- TURNER, Ashley, et al. Personal exposure to average weekly ultrafine particles, lung function, and respiratory symptoms in asthmatic and non-asthmatic adolescents. Environment international, 2021, 156: 106740

\section{Inquinamento da sostanze chimiche non atmo- sferiche}

1. Consensus italiana sulla gestione clinica e terapeutica della 'Sensibilità chimica multipla' (MCS, Multiple Chemical Sensitivity)

In questo articolo gli autori pubblicano la prima Consensus di 
esperti italiana e una tra le poche a livello mondiale sulla 'Sensibilità multipla a sostanze chimiche' (MCS). Tale entità nosologica viene definita come una affezione multisistemica, ricorrente, di origine ambientale, con esacerbazioni periodiche in risposta a diverse sostanze (pesticidi, solventi, metalli tossici e muffe). La diagnosi si pone in presenza di tutti i seguenti sei criteri:

1. condizione di durata $>6$ mesi con scadimento della qualità di vita e di funzioni organiche;

2. sintomi ricorrenti e riproducibili che coinvolgono anche il sistema nervoso, con una caratteristica ipersensibilità agli odori;

3. sintomi che coinvolgono il SNC + un altro sintomo;

4. risposta riproducibile a triggers presenti in bassa concentrazione;

5. miglioramento o risoluzione dei sintomi dopo rimozione del trigger.

Gli autori (allergologi, dermatologi, reumatologi, anestesisti, dentisti, ORL e biologi) hanno effettuato una revisione sistematica (PubMed e EMBASE) delle evidenze cliniche e terapeutiche disponibili sulla MCS. Nell'articolo vengono approfonditi gli esami di primo e secondo livello consigliati, divisi per apparato (cardiovascolare, neurologico, endocrinologico, reumatologico etc). É disponibile un questionario validato per lo screening della MCS: BREESI (Brief Environmental Exposure and Sensitivity Inventory), i pazienti positivi a tale questionario dovranno essere sottoposti al questionario diagnostico validato QEESI (Quick Environmental Exposure and Sensitivity Inventory). L'obiettivo della Consensus è diffondere informazioni sulla MCS tra medici e tra i pazienti e chiarire eventuali diagnosi errate di MSC non basate sulle evidenze.

- DAMIANI, Giovanni, et al. Italian Expert Consensus on Clinical and Therapeutic Management of Multiple Chemical Sensitivity (MCS). International Journal of Environmental Research and Public Health, 2021, 18.21: 11294

\section{Esposizione materna agli ftalati e valori pressori durante e dopo la gravidanza nello studio PROGRESS}

Gli ftalati possono interferire con i metaboliti che regolano la pressione arteriosa. In considerazione dei cambiamenti profondi della vascolarizzazione durante la gravidanza, le donne in gravidanza possono essere particolarmente suscettibili a questi effetti. Nel presente lavoro gli autori hanno esaminato l'esposizione agli ftalati durante la gravidanza e monitorato i valori pressori da metà gravidanza fino a 72 mesi dopo il parto in 892 donne con gravidanze singole. Le donne sono state arruolate all' interno dello studio di coorte PROGRESS (un programma di ricerca su obesità, crescita, ambiente e fattori di stress sociale condotto a Città del Messico). 15 metaboliti degli ftalati sono stati dosati in campioni urinari raccolti nel II e III trimestre. La misura delle pressione ed altri dati sono stati raccolti nel corso di 9 visite fino a 72 mesi dopo il parto. Gli autori hanno utilizzato diversi metodi statistici (modelli lineari, misti, di regressione) per stimare la relazione tra la concentrazione di ftalati urinari ed i valori di pressione arteriosa. I risultati hanno evidenziato che come miscela complessiva le concentrazioni dei biomarcatori degli ftalati erano associate ad un aumento dei valori pressori durante il II e III trimestre gestazionale. Per quanto riguarda i singoli biomarcatori le concentrazioni nel secondo trimestre di monobenzil ftalato (MbzP) e di 2 EtilexilFtalato (RDEHP) erano associate con valori più alti di pressione nel III trimestre. Successive valutazioni statistiche (LCGMs) hanno identificato due diverse classi di traiettorie pressorie: una prima caratterizzata da un incremento consecutivo fino a 72 mesi dopo il parto ed una seconda con un decremento fino a 18 mesi dopo il parto e poi un successivo incremento. L'esposizione ad una miscela di ftalati durante la gravidanza era associata ad un maggiore probabilità di essere nella prima classe (ovvero incremento persistente) e simili associazioni sono state viste anche per altri due biomarcatori degli ftalati quali mono2etil5carbossipentilterftalato (MECPTP) e di dibutilftalato, osservando relazioni temporali specifiche per tutti i biomarcatori sopracitati. In sintesi le donne di questa coorte esposte agli ftalati e ai loro biomarcatori presentavano valori pressori più alti nella parte terminale della gravidanza oltre ad un cambiamento a lungo termine nelle traiettorie della pressione sanguigna.

- WU, Haotian, et al. Maternal phthalates exposure and blood pressure during and after pregnancy in the PROGRESS study. Environmental health perspectives, 2021, 129.12: 127007

\section{Esposizione prenatale a miscele chimiche non persistenti e crescita fetale: uno studio di popolazione}

L'esposizione prenatale a sostanze chimiche non persistenti è universale. L'effetto di questa esposizione complessiva sulla crescita fetale è stato poco analizzato finora, in quanto la maggior parte degli studi si sono limitati a modelli di esposizione singola, ignorando il loro potenziale impatto cumulativo. Gli autori di questo studio hanno cercato di valutare l'associazione tra l'esposizione prenatale a una miscela di ftalati, bisfenoli e pesticidi organofosfati (OP) e la crescita fetale, controllando le misure della circonferenza cranica, della lunghezza del femore e del peso. Si è trattato di uno studio di coorte effettuato nei Paesi Bassi su 776 gravidanze. L'esposizione è stata stimata misurando in varie fasi della gravidanza le concentrazioni urinarie di 11 metaboliti degli ftalati, 3 bisfenoli e 5 metaboliti del dialchilfosfato (DAP). Gli esiti sono stati valutati mediante misure ecografiche della circonferenza della testa, della lunghezza del femore e del peso fetale stimato (PFS) raccolte tra 18-25 settimane di gravidanza e dopo le 25 settimane, e mediante misurazioni della circonferenza della testa, della lunghezza e del peso effettuate alla nascita. Il PFS medio tra le 18-25 settimane e oltre le 25 settimane era 369 e 1626 grammi rispettivamente, e il peso medio alla nascita era 3451 grammi. Una maggiore esposizione alle sostanze chimiche è stata associata a parametri di crescita fetali e neonatali inferiori, anche se in modo non lineare. A 18-25 settimane, i feti nel secondo, terzo e quarto quartile di esposizione (Q2-Q4) pesavano 26 g [95\% CI: 38-13], 35g (95\% CI:55-15), e 27g (95\% CI: 54, 1) meno rispetto a quelli del primo quartile (Q1). Un modello dose-risposta simile è stato osservato oltre le 25 settimane di gravidanza, ma con differenze inferiori. Alla nascita infine non si sono osservate differenze di peso tra Q1-Q2 o Q1-Q3. Tuttavia, i feti maggiormente esposti (Q4) pesavano 91g (95\% CI: -258, 76) meno rispetto a quelli del Q1. I risultati osservati a 18-25 settimane e oltre le 25 settimane di gravidanza per la lunghezza del femore erano simili ma queste differenze non si sono confermate alla nascita. Infine non sono state osservate variazioni significative nella circonferenza cranica. Queste osservazioni, secondo gli autori, dimostrano che livelli elevati di esposizione cumulativa a 
queste sostanze possono influenzare il peso alla nascita, ed avere quindi un impatto negativo sulla crescita fetale.

- VAN DEN DRIES, Michiel A., et al. Prenatal Exposure to Nonpersistent Chemical Mixtures and Fetal Growth: A Population-Based Study. Environmental health perspectives, 2021, 129.11: 117008

\section{Esposizione ai rifiuti elettronici e salute: una revisione si- stematica}

I rifiuti elettronici (e-waste) contengono numerose sostanze chimiche dannose per la salute umana. Gli autori di questo articolo hanno aggiornato una revisione già pubblicata nel 2013 che analizzava le conseguenze negative per la salute umana dell'esposizione ai rifiuti elettronici. Sono stati cercati gli articoli pubblicati in lingua inglese tra il 18 dicembre 2012 e il 28 gennaio $2020 \mathrm{su}$ emBASE, PsycNET, Web of Science, CINAHL e PubMed. Sono stati identificati 5645 record, di cui 70 soddisfacevano i criteri preimpostati e sono stati quindi analizzati. La maggior parte degli studi sono stati condotti in Cina $(n=66)$, seguita da Vietnam $(n=2)$, Ghana $(n=1)$ e India $(n=1)$. Le persone che vivono nelle aree esposte ai rifiuti elettronici avevano livelli ematici significativamente elevati di metalli pesanti (piombo, cadmio, mercurio, manganese, cromo, nichel) e inquinanti organici persistenti: IPA, PBDE, bifenoli policlorurati (PCB), PCDD, nuovi ritardanti di fiamma (NFR), bifenili polibromurati, esteri di ftalati, bisfenoli e organofosfati. Segnaliamo i principali effetti sulla salute emersi dalla revisione, rimandando all'articolo completo per una analisi più approfondita. L'aumento dei livelli di sostanze tossiche nel periodo prenatale è stato associato a uno scarso sviluppo fetale nei primi anni di vita. Uno studio ha riportato che la concentrazione ematica degli IPA nei neonati era negativamente associata all'altezza e alla circonferenza del torace nei bambini di età compresa tra 3 e 7 anni. In tre studi l'esposizione al piombo è stata associata a una diminuzione della crescita e dello sviluppo del bambino. Livelli più elevati di piombo nel sangue sono stati associati a una limitazione dello sviluppo neurologico. L'esposizione a sostanze chimiche tossiche indotte dai rifiuti elettronici modifica la funzione tiroidea e ha effetti di interferenza endocrina sugli ormoni sessuali. I risultati di due studi hanno osservato una alterazione dei telomeri tra le donne in gravidanza esposte professionalmente ai rifiuti elettronici e tra i residenti. I dati hanno suggerito che le alterazioni telomeriche sono potenzialmente indotte dall'esposizione al cadmio e agli inquinanti organici persistenti (POP). Nel complesso, i risultati suggeriscono anche che l'esposizione ai rifiuti elettronici è associata a un elevato stress ossidativo. Gli esiti respiratori e cardiovascolari infine sono stati studiati in numerosi studi in Cina, dove i dati suggeriscono che vivere in un'area esposta ai rifiuti elettronici potrebbe aggravare i sintomi respiratori dei bambini di età compresa tra 2 e 8 anni. Cinque studi hanno riportato esiti cardiovascolari correlati all'esposizione ai rifiuti elettronici, con aumento dei livelli di sostanze chimiche tossiche e concomitanti misure anomale della fisiologia cardiovascolare. Gli autori di questa revisione confermano che le sostanze chimiche tossiche presenti nei rifiuti elettronici possono avere un impatto negativo significativo sulla salute delle persone che vivono in aree esposte, in particolare durante le finestre sensibili dello sviluppo come la gravidanza e l'infanzia. Nei siti di rifiuti elettronici devono essere impiegati nuovi metodi efficaci in termini di costi per operazioni di riciclaggio sicure per garantire la salute e la sicurezza delle popola- zioni vulnerabili.

- PARVEZ, Sarker M., et al. Health consequences of exposure to e-waste: an updated systematic review. The Lancet Planetary Health, 2021, 5.12: e905-e920

5. Aumento di prevalenza di dermatite atopica in bambini di età compresa tra 0-3 anni altamente esposti ai parabeni Negli ultimi anni si è assistito ad un notevole incremento delle allergopatie, tra cui la dermatite atopica, e diverse ipotesi sono state elaborate al fine di spiegare questo andamento. Tra queste, l'"Hygiene Hypothesis" mette in evidenza il ruolo svolto da agenti antimicrobici e conservanti nel contribuire allo sviluppo di allergie nelle comunità altamente sviluppate. L'esposizione ai parabeni, contenuti in moltissimi prodotti di igiene come saponi, shampoo, cosmetici, è già stata studiata in correlazione alla comparsa di asma e di dermatite da contatto. Questo recente studio giapponese ha studiato tra il 2017 e il 2019 la relazione tra esposizione ai parabeni e la prevalenza di dermatite atopica in 236 bambini di età compresa tra 0 e 3 anni, fascia d'età precedentemente mai studiata, attraverso l'analisi della concentrazione di parabeni nelle urine. I risultati ottenuti mostrano la presenza di alte concentrazioni di parabeni urinari nei soggetti con dermatite atopica $(n=31)$, senza differenze per sesso ed età, rispetto ai controlli sani $(n=205)$, con associazione statisticamente significativa $(\mathrm{p}<0.001)$, anche dopo aggiustamenti per fattori confondenti (sesso, età fumo passivo). Tuttavia, sono necessari studi longitudinali numericamente più ampi e con una valutazione più dettagliata della dermatite atopica e dosaggi seriati dei parabeni urinari per confermare i dati ottenuti.

- ARAFUNE, Johichi, et al. Increased Prevalence of Atopic Dermatitis in Children Aged 0-3 Years Highly Exposed to Parabens. International journal of environmental research and public health, 2021, 18.21: 11657

\section{Agricoltura di città, benefici e rischi}

L'agricoltura urbana consiste nel coltivare, trasformare e distribuire il cibo all'interno di contesti urbanizzati o peri-urbani, come città e villaggi. Diverse evidenze scientifiche ne sottolineano i benefici sociali, economici, ambientali e di salute. Tuttavia esistono pochi studi che abbiano analizzato il rischio di contaminazione da metalli per chi lavora in questo settore e per i consumatori. L'obiettivo di questo studio, che ha visto la collaborazione di diversi partner della comunità (privi di conflitti d'interesse) è stato rispondere alle domande emerse dai cittadini in merito alla sicurezza dell'agricoltura urbana e dei prodotti derivanti da questa pratica. Sono state misurate le concentrazioni di 9 metalli, 6 non essenziali (arsenico, bario, cadmio, cromo, piombo, nichel) e 3 essenziali (rame, manganese e zinco) nel suolo, nelle acque di irrigazione e in 13 diversi tipi di ortaggi coltivati da 104 diversi luoghi di agricoltura urbana nell'area di Baltimora. Le misurazioni ottenute sono state confrontate con le linee guida prodotte dal dipartimento di salute pubblica e dove queste non fossero disponibili i dati sono stati confrontati con studi eseguiti in zone rurali. I dati finali della ricerca suggeriscono che l'agricoltura urbana è sicura per quanto riguarda l'esposizione ai metalli. In particolare è emerso che le concentrazioni dei metalli nelle acque di irrigazione sono inferiori ai valori considerati validi per le acque potabili. Per quanto concerne il suolo i valori sono al di sotto 
dei valori soglia per bario, cadmio, piombo e nichel mentre sono risultati borderline per arsenico e cromo, comunque sicuri. La valutazione più complessa spetta ai prodotti agricoli dove è risultata una minima differenza di concentrazione dei metalli statisticamente significativa tra quelli coltivati nelle aree urbane o non urbane ma di difficile interpretazione. Ad esempio è stata trovata una concentrazione aumentata di arsenico, bario e piombo nella verdura a foglia coltivata in zone urbane rispetto a quella coltivata in aree non urbane, nonostante i livelli di piombo segnalati fossero di molto inferiori a quelli raccomandati dall'OMS. Gli autori concludono che sono necessari approfondimenti in questo ambito per poter dare significato a questi valori. In conclusione la pratica dell'agricoltura urbana è da consigliare parallelamente alla realizzazione di linee guida, prodotte dai dipartimenti di sanità pubblica, in grado di stabilire i limiti di sicurezza di esposizione ai metalli del sottosuolo, delle acque di irrigazione e dei prodotti agricoli. Gli autori inoltre suggeriscono di approfondire anche i potenziali benefici relativi all'assunzione di metalli essenziali nei prodotti ottenuti dall'agricoltura urbana.

LUPOLT, Sara N., et al. The Safe Urban Harvests Study: A Community-Driven Cross-Sectional Assessment of Metals in Soil, Irrigation Water, and Produce from Urban Farms and Gardens in Baltimore, Maryland. Environmental health perspectives, 2021, 129.11: 117004

\section{Assunzione di farmaci nitrosabili, apporto di nitrato con l'acqua potabile e rischio di natimortalità in una coorte di donne danesi}

Si stima che il 15-24\% delle donne statunitensi e danesi usi un farmaco nitrosabile durante la gravidanza, tra cui antibiotici, farmaci per l'asma e antiemetici. Molti farmaci infatti contengono composti come ammine secondarie e terziarie o ammidi. Il Nitrato (NO3-) contenuto normalmente nell' acqua potabile o derivante da altre fonti viene convertito in nitrito (NO2-) nel corpo umano e successivamente può quindi reagire con ammine e ammidi nel tratto gastrointestinale e formare in ambienti acidi come nello stomaco composti N-nitroso (NOC) altamente teratogeni. I NOC si formano in misura maggiore se la concentrazione di agente nitrosante è elevata e la loro formazione nello stomaco materno con successiva trasmissione transplacentare al feto ne rappresenta la fonte più probabile di esposizione prenatale. L'effetto teratogeno dei NOC è legato a processi di alchilazione del DNA. È noto come l'esposizione prenatale ai farmaci nitrosabili possa causare malformazioni congenite e parto pretermine, ma pochi studi hanno esaminato la relazione tra la loro assunzione e la natimortalità. Scopo dello studio era dimostrare un rischio di eventi avversi (nati morti) superiore nelle donne che avevano assunto un farmaco nitrosabile durante la gravidanza e la cui acqua potabile contenesse elevate concentrazioni di NO3 (>25 mg/L) rispetto a gravide che non avessero assunto tali farmaci in gravidanza. È stato dunque condotto uno studio di coorte su 652.810 donne primigravide presenti nel registro danese delle nascite tra il 1997 e il 2017. La popolazione è stata divisa in un gruppo di donne che avevano ricevuto un farmaco nitrosabile prima della $22^{\circ}$ settimana e in un gruppo di riferimento che non aveva assunto tali farmaci in gravidanza. Dallo studio è emerso che la sola esposizione ai nitrati dell'acqua potabile non era associata al rischio di natimortalità. Tra le 100.244 donne che avevano assunto un farmaco nitrosabile prima della $22^{\wedge}$ settima- na gestazionale, lo $0.42 \%$ (418) hanno avuto un nato morto rispetto allo $0.36 \%$ tra le 552.566 donne del gruppo di riferimento. Donne che avevano assunto farmaci con componenti nitrosabili e con concentrazioni di nitrato comprese nell'intervallo $>1$ ed $\leq 2$ $\mathrm{mg} / \mathrm{l}$ nell'acqua potabile avevano invece un aumentato rischio di nati morti (RR 1.55, intervallo di conversazione al 95\%, 1.152.09) rispetto alle donne del gruppo di riferimento. Dall'analisi stratificata è emerso che il più alto rischio di evento avverso (nati morti) è stato riscontrato tra le donne con assunzione secondaria di ammine e concentrazioni di nitrato $>25 \mathrm{mg} / \mathrm{L}$ nell'acqua domestica (RR 3.11, IC 95\%, 1.08-8.94). In conclusione l'associazione tra l'assunzione di farmaci nitrosabili e il rischio di natimortalità può dipendere dal livello di nitrati presenti nell'acqua potabile domestica.

THOMSEN, Anne Marie Ladehoff, et al. Prenatal nitrosatable prescription drug intake, drinking water nitrate, and the risk of stillbirth: a register-and population-based cohort of Danish pregnancies, $1997-$ 2017. Environmental Health, 2021, 20.1: 1-10. -z

\section{Esposizione agli ftalati e tratti autistici nell'infanzia}

Diversi studi di correlazione fra esposizione agli ftalati e comparsa di tratti autistici nei bambini hanno finora mostrato risultati incoerenti rispetto al sesso e alle finestre di suscettibilità. Gli autori di questo lavoro hanno studiato l'associazione fra esposizione agli ftalati durante il periodo prenatale e l'infanzia con la comparsa di tratti autistici utilizzando dati provenienti da una coorte di nascita coreana. Sono state valutate 547 coppie madre-figlio in cui sono stati misurati cinque metaboliti degli ftalati durante la gravidanza e all'età di 4, 6 e 8 anni. Per valutare la comparsa di tratti autistici è stato utilizzato il questionario SCQ (Social Communication Questionnaire), che valuta le capacità di comunicazione dei bambini, il funzionamento sociale e i comportamenti ripetitivi negli ultimi tre mesi. I punteggi sono poi stati analizzati in base alle finestre di esposizione e al sesso. I risultati hanno evidenziato come un aumento di 2.7 volte dei livelli del metabolita del di-(2-etilesile) ftalato, mono-(2-etil-5-idrossiesile) ftalato (MEHHP) e mono-(2-etil-5-ossoesil) ftalato (MEOHP) durante la gravidanza fosse associato ad un aumento dei punteggi SCQ dell' $8.5 \%$, solo all'età di 4 anni. Inoltre, i livelli di MEHHP all'età di 4 e 8 anni sono risultati associati ad un aumento dei punteggi SCQ rispettivamente del 9.9\% e del 9.6\%. Si sono inoltre evidenziate associazioni più forti tra esposizione agli ftalati e punteggi SCQ nel sesso maschile rispetto alle femmine. Gli autori concludono come lo studio indichi diverse possibili finestre temporali suscettibili di esposizione agli ftalati: l'esposizione durante la gravidanza sembra associata a tratti autistici nei bambini piccoli, mentre l'esposizione durante la prima infanzia sembra portare alla comparsa di tratti autistici nei bambini in età scolare, in particolare nel sesso maschile.

${ }^{\circ} \mathrm{KIM}$, Johanna Inhyang, et al. Association of phthalate exposure with autistic traits in children. Environment International, 2021, 157: 106775

\section{I composti per e polifluoralchilici (PFAS) alterano la com- posizione lipidica del latte materno}

Gli autori di questo studio hanno cercato di definire l'impatto dell'esposizione materna ad alcuni PFAS sulla composizione lipidica del latte materno ed inoltre hanno studiato l'impatto 
combinato dell'esposizione a PFAS sulla composizione lipidica del latte materno e sulla crescita dei bambini. In 44 coppie madre-bambino sono stati misurati i livelli di PFAS e lipidi nel siero materno ed è stata condotta l'analisi della composizione lipidica del latte materno raccolto 2-4 giorni dopo il parto e a 3 mesi di età. Come biomarcatori gastrointestinali sono state misurate la calprotectina e la betadefensina 2 umana su campioni di feci dei bambini all'età di 3, 6, 9 e 12 mesi. La dieta materna è stata valutata con un questionario validato. I risultati hanno evidenziato che i livelli di PFAS erano inversamente associati ai livelli di lipidi totali nel latte materno raccolto dopo il parto. Nel gruppo ad alta esposizione, il rapporto fra gli acidi grassi saturi e polinsaturi acilati nei triacilgliceroli era aumentato. Inoltre, un'elevata esposizione a PFAS è risultata associata con un'alterata composizione fosfolipidica, indicativa di un aumento sfavorevole della dimensione dei globuli di grasso del latte. Questi cambiamenti nella composizione lipidica del latte sono stati ulteriormente associati a una più lenta crescita nei bambini e al riscontro di elevati livelli di marcatori infiammatori intestinali. I dati suggeriscono come l'esposizione materna a PFAS possa influenzare la qualità nutrizionale del latte materno, cosa che, a sua volta, può avere un impatto negativo sulla salute e sulla crescita dei bambini nelle epoche di vita successive.

- LAMICHHANE, Santosh, et al. Exposure to per-and polyfluoroalkyl substances associates with an altered lipid composition of breast milk. Environment International, 2021, 157: 106855

\section{Esposizione prenatale e postnatale ai PFAS, fattori car- diometabolici e stato di infiammazione nei bambini di 6 co- orti europee}

Lo studio ha ipotizzato che l'esposizione precoce ai PFAS sia associata ad una ridotta salute metabolica nei bambini. È stata analizzata l'associazione tra l'esposizione prenatale e postnatale ai PFAS e la salute cardiometabolica analizzando anche il ruolo delle proteine infiammatorie. È stata misurata la concentrazione dei PFAS nel sangue delle donne in gravidanza e dei bambini all'età di 8 anni considerando 1101 coppie madre-figlio. Si è poi stimata l'associazione tra l'esposizione ai PFAS e alcuni fattori cardiometabolici come la circonferenza vita (WC), la pressione sanguigna sistolica e diastolica (BP), la concentrazione dei trigliceridi (TG), delle lipoproteine ad alta densità (HDL-C) e a bassa densità (LDL-C). Sono stati inoltre misurati 36 biomarcatori infiammatori nel plasma dei bambini coinvolti. E emerso che l'esposizione ai PFAS è associata positivamente ai livelli di HDL-C e di pressione arteriosa mentre è associata negativamente a WC, LDL-C e TG. Risultati diversi si sono osservati con i singoli PFAS e non considerando la miscela di PFAS; per esempio nel caso di esposizione prenatale a $\mathrm{PFHxS}$ si è osservata un'associazione negativa con HDL-C mentre con l'esposizione prenatale a PFNA si è osservata unassociazione positiva con WC. Questi ultimi dati sono in contrasto con quelli ottenuti considerando la miscela di PFAS. Inoltre i biomarcatori infiammatori sono risultati positivamente associati ai diversi fattori cardiometabolici analizzati e solo l'esposizione prenatale a PFOA è risultata positivamente associata ai livelli della citochina pro-infiammatoria IL-1.

- PAPADOPOULOU, Eleni, et al. Prenatal and postnatal exposure to PFAS and cardiometabolic factors and inflammation status in children from six European cohorts. Environment International, 2021, 157:
106853

11. Glifosato e acido aminometilfosfonico (AMPA) nelle urine di bambini ed adolescenti in Germania: uno studio di biomonitoraggio

Questo studio ha analizzato l'esposizione al glifosato in una coorte di bambini e adolescenti in Germania. Sono stati analizzati 2144 campioni di urine di bambini e adolescenti (età compresa tra i 3 e i 17 anni). Nel 52\% dei campioni (e nel 46\% considerando il metabolita AMPA) la concentrazione urinaria di glifosato è risultata essere superiore al limite di quantificazione $(0.1 \mu \mathrm{g} / \mathrm{L})$. La concentrazione media è risultata di $0.107 \mu \mathrm{g} / \mathrm{L}(0.090 \mu \mathrm{g} / \mathrm{g}$ creatinina) per il glifosato e di $0.100 \mu \mathrm{g} / \mathrm{L}(0.085 \mu \mathrm{g} / \mathrm{g}$ creatinina $)$ per AMPA. Non è stata evidenziata una chiara associazione tra l'esposizione a tali sostanze e una dieta vegetariana o un consumo di cereali, legumi o verdure. I bassi livelli di quantificazione sia per il glifosato che per il suo metabolita hanno evidenziato che complessivamente, nella popolazione analizzata, l'esposizione è bassa.

' LEMKE, Nora, et al. Glyphosate and aminomethylphosphonic acid (AMPA) in urine of children and adolescents in Germany-Human biomonitoring results of the German Environmental Survey 2014-2017 (GerES V). Environment International, 2021, 156: 106769

\section{Esposizione perinatale a potenziali interferenti endocrini e rischio di criptorchidismo in Norvegia}

L'obiettivo di questo studio prospettico è stato quello di valutare 27 potenziali EDCs (interferenti endocrini) misurati nel latte materno come indice di esposizione perinatale e il rischio di criptorchidismo. Sono stati considerati 641 bambini maschi a cui erano stati misurati, nel latte materno, 27 EDCs (5 pesticidi organoclorurati, 14 bifenili policlorurati (PCBs), 6 ritardanti di fiamma bromurati e 2 sostanze poli e perfluoroalchiliche). Sono stati definiti i casi di criptorchidismo congenito, ricorrente, persistente e sempre presente basandosi su questionari sottoposti alle madri quando i bambini avevano 1, 6, 12 e 24 mesi. È emerso che la prevalenza di criptorchidismo congenito era del $6.1 \%$ e nella metà dei casi si è assistito a una discesa spontanea del testicolo entro i 6 mesi di vita, dopo i quali la prevalenza si è stabilizzata tra il 2.2 e il $2.4 \%$. La prevalenza di criptorchidismo sempre riportato a 1, 6, 12 e 24 mesi era del 12.2\%. I migliori predittori di criptorchidismo sono risultati essere il PCB-74, il PCB-114, il PCB-194 e il $\beta$-HCH. Tali sostanze sono associate a un rischio aumentato di presentare criptorchidismo congenito. Alcuni PCBs possono risultare falsamente associati a criptorchidismo se considerati individualmente per il fatto che può esserci unesposizione contemporanea ad altre sostanze chimiche correlate. Sono necessari ulteriori studi per confermare i risultati osservati.

- DESALEGN, Anteneh Assefa, et al. A case-cohort study of perinatal exposure to potential endocrine disrupters and the risk of cryptorchidism in the Norwegian HUMIS study. Environment International, 2021, 157: 106815

\section{Associazione tra una miscela di fenoli e ftalati e compor- tamento del bambino}

Questo studio di coorte francese ha analizzato l'effetto combinato dell'esposizione prenatale ai fenoli e agli ftalati e il comportamento dei bambini utilizzando misure di esposizione ri- 
petuta. Sono stati misurati 12 fenoli, 13 ftalati e 2 plastificanti senza ftalati in multipli campioni di urina raccolti da madri in gravidanza (416 coppie madre-figlio della coorte francese SEPAGES). Il comportamento infantile è stato valutato a due anni di età considerando la Checklist Child Behaviour 1.5-5 (CBCL). Utilizzando la regressione dei quantili è stata analizzata l'associazione tra l'esposizione a una miscela dei composti analizzati e i disturbi internalizzanti ed esternalizzanti nei bambini. È emerso che l'esposizione è associata a maggior rischio sia dei disturbi internalizzanti che esternalizzanti in tutta la popolazione analizzata. È emersa inoltre unassociazione più marcata nelle bambine rispetto ai bambini. In quest'ultime l'associazione si è verificata in particolare con il bisfenolo A, il triclosano e con lo ftalato monoetilico per i disturbi esternalizzanti mentre con lo ftalato monoetilico, il monobenzil ftalato e lo ftalato mono-n-butile per i disturbi internalizzanti.

- GUILBERT, Ariane, et al. Associations between a mixture of phenols and phthalates and child behaviour in a French mother-child cohort with repeated assessment of exposure. Environment International, 2021, 156: 106697

\section{Psicologia ambientale}

1. Effetti a breve e lungo termine degli incentivi economici sulla volontà di utilizzare i mezzi pubblici per recarsi al lavoro Si tratta di uno studio longitudinale eseguito per valutare gli effetti di un incentivo economico sulla volontà e sulle motivazioni alla base dell'utilizzo dei mezzi pubblici per recarsi al lavoro sul breve e sul lungo periodo (quindi anche dopo che l'incentivo era stato sospeso). I criteri di inclusione erano: essere residenti entro $20 \mathrm{~km}$ dalla città di Groningen e l'uso abituale dell'auto per andare al lavoro. La persone ricevevano un ticket gratuito per il tragitto in autobus casa-lavoro, valido 3 settimane. I partecipanti compilavano un questionario prima, subito dopo e a tre mesi di distanza dall'utilizzo della card gratuita. Veniva valutata la motivazione per la quale sceglievano i trasporti pubblici, l'importanza economica, simbolico-sociale, ambientale, di accessibilità urbana che spingeva al loro utilizzo, l'intenzione di continuare ad usarli. I partecipanti in numero di 380 avevano risposto al primo questionario, 69 anche al secondo e 17 a tutti e tre. L'incentivo economico era efficace nel favorire l'uso dei mezzi pubblici quando era in atto, ma dopo la sua sospensione non veniva mantenuta l'abitudine di andare al lavoro con tali mezzi. L'importanza economica era presente quando l'incentivo veniva erogato, quella ambientale, di gratificazione sociale e di accessibilità urbana non variavano dopo lo studio, probabilmente perché i partecipanti non scoprivano alcun ulteriore vantaggio nell'utilizzare i mezzi. La motivazione era invariata pre e post test, per tale ragione gli autori propongono di selezionare maggiormente le categorie sensibili al tema ambientale per favorire e rafforzare un comportamento virtuoso al quale la persona è già predisposta. Gli autori propongono inoltre di effettuare ulteriori studi informando maggiormente le persone sui benefici ambientali (es. CO2 risparmiata), o gratificandole socialmente nell'utilizzo dei mezzi pubblici (es. con la finalità di contribuire a ridurre l'inquinamento), per ottenere altre motivazioni durature che spingano all'utilizzo dei mezzi dopo che l'incentivo economico si è esaurito. I limiti dello studio sono l'elevato drop out partecipanti, l'assenza di gruppo controllo, il fatto che esso valuta le intenzioni, non i comportamenti in atto.

${ }^{\circ}$ ZEISKE, Nadja; VAN DER WERFF, Ellen; STEG, Linda. The effects of a financial incentive on motives and intentions to commute to work with public transport in the short and long term. Journal of Environmental Psychology, 2021, 78: 101718

2. Comprendere le intenzioni dei genitori di consentire ai loro figli di andare a scuola a piedi o in bicicletta: il questionario PILCAST

I bambini in genere non soddisfano la raccomandazione di eseguire 60 minuti di attività fisica al giorno e lo spostamento per andare a scuola può essere un'opportunità per incrementare il loro livello di attività fisica. È fondamentale coinvolgere i genitori per raggiungere questo obiettivo. Studi precedenti hanno mostrato che la maggior parte delle barriere che i genitori pongono allo spostamento a piedi dei loro bambini per raggiungere la scuola sono la sicurezza stradale, la sicurezza dell'ambiente e la distanza dalla scuola; molti genitori inoltre ritengono la bicicletta più pericolosa del camminare, e vi sono differenze significative tra le due modalità in vari paesi europei (es. l'uso della bicicletta è maggiore in Danimarca rispetto al Regno Unito). Secondo gli autori di questo studio vi è la necessita di comprendere meglio quali siano i fattori che i genitori percepiscono come facilitanti al fine di promuovere il cambiamento di comportamento per quanto riguarda lo spostamento attivo a scuola (AST Active School Transportation). Gli autori hanno quindi utilizzato il questionario PILCAST basato sulla teoria del comportamento pianificato (TBP Theory of Planned Behavior) per comprendere la predisposizione dei genitori nel lasciar andare i loro bambini a scuola a piedi o in bicicletta. La TBP è un modello che spiega il comportamento umano ed è basato sulla interazione di intenzione, atteggiamento, norme soggettive e percezione del controllo (ovvero la percezione che un individuo ha di poter mettere in atto il comportamento voluto) ed è stato molto utilizzato nella psicologia del traffico. Gli autori hanno raccolto 1.024 risposte da parte dei genitori e l'analisi di regressione mostrava che la TBP spiegava il 55.3\% delle intenzioni dei genitori di lasciar andare i figli a scuola in bicicletta e il $20.6 \%$ per quanto riguarda lo spostamento a piedi; nello specifico un ruolo importante era svolto dalle abitudini pregresse dei genitori ed i fattori che influenzano maggiormente l'andare a scuola in bicicletta erano il controllo percepito del comportamento, l'attitudine positiva, le norme soggettive (ovvero la percezione morale) oltre alle norme descrittive (ovvero quello che i membri di un gruppo fanno in una data situazione) e per l'andare a scuola a piedi soprattutto le norme soggettive e descrittive. Secondo gli autori il questionario PILCAST è utile per capire la predisposizione psicologica e gli antecedenti che possono influenzare la decisione dei genitori nel lasciar andare i figli a scuola in bicicletta o a piedi. Potrebbe quindi fornire informazioni importanti per incentivare queste modalità di spostamento e di conseguenza fare in modo che $\mathrm{i}$ bambini facciano più attività fisica.

${ }^{\circ}$ FORSBERG, Hanna, et al. Development and Initial Validation of the PILCAST Questionnaire: Understanding Parents' Intentions to Let Their Child Cycle or Walk to School. International journal of environmental research and public health, 2021, 18.21: 11651 
3. I bambini inconsapevoli consumatori di carne: una opportunità per affrontare il cambiamento climatico

Gli autori partono dalla riflessione su come una dieta a base vegetale sia ad oggi uno dei modi più efficaci con cui si può ridurre la propria impronta ambientale. Tuttavia il consumo globale di carne e altri prodotti animali risulta in costante aumento. Studiare cosa pensano i bambini riguardo al cibo potrebbe, secondo gli autori, fare luce sul rapporto tra comportamenti alimentari della popolazione e cambiamento climatico. Sono state valutate le conoscenze dei bambini riguardo all'origine (vegetale o animale) degli alimenti, nonché la loro opinione su cosa si possa o non si possa mangiare. Il campione era composto da bambini di età compresa tra 4 e 7 anni provenienti dagli Stati Uniti. Ai bambini è stato richiesto di dividere le immagini di alimenti, che erano loro fatte visionare, in quelli di origine vegetale e quelli di origine animale e in una secondo set di immagini di dividere quelli commestibili da quelli non commestibili. I risultati hanno mostrato diversi errori nelle conoscenze di base dei bambini sulle origini del cibo. Gli errori più frequenti riguardavano gli alimenti derivati dagli animali (soprattutto, ma non esclusivamente carni). Da questa considerazione deriva necessariamente una serie di domande incentrata su come i bambini possano accettare messaggi apparentemente contraddittori sul trattare gli animali con gentilezza e su pratiche alimentari che richiedono la macellazione degli animali per "trasformarli" in cibo (ad esempio sembra che alcuni bambini pensino che la carne venga raccolta da animali che sono morti per cause naturali). Gli autori concludono come i bambini, che erediteranno inevitabilmente la crisi climatica perpetrata dalle generazioni precedenti, dovrebbero essere visti come agenti di cambiamento ambientale. Anche se non votano o ricoprono incarichi di leadership, possiedono atteggiamenti e giudizi che supportano comportamenti rispettosi (o irrispettosi) dell'ambiente. L'infanzia può quindi rappresentare un'occasione unica durante la quale stabilire abitudini alimentari per tutta la vita che aiutino a mitigare il cambiamento climatico.

${ }^{\circ}$ HAHN, Erin R.; GILLOGLY, Meghan; BRADFORD, Bailey E. Children are unsuspecting meat eaters: An opportunity to address climate change. Journal of Environmental Psychology, 2021, 78: 101705

\section{Le reazioni agli avvisi riguardanti il cambiamento climati- co nella popolazione}

La popolazione riceve quotidianamente comunicazioni e notizie sul rischio di raggiungere un punto critico di cambiamento climatico che porti a conseguenze irreversibili. Tuttavia, si conosce poco su come tali avvisi possano promuovere un cambiamento comportamentale o su come le emozioni possano essere alla base di tali cambiamenti. Gli autori ci illustrano due esperimenti online preregistrati attraverso i quali è stata studiata la reazione delle persone in risposta ad informazioni circa la criticità della situazione ambientale utilizzando una modalità di gioco online. I risultati hanno mostrato come il senso di colpa sia costantemente associato al cambiamento comportamentale. Più specificamente il senso di colpa sembrerebbe mediare l'effetto che gli avvertimenti sull'ambiente hanno sul cambiamento comportamentale, il che significa che livelli più elevati di colpa sono predittivi di un più incisivo cambiamento comportamentale. I risultati sottolineano il ruolo della colpa come percorso affettivo verso l'effettivo cambiamento di comportamento pro-ambientale. Il senso di colpa (con vergogna, imbarazzo e orgoglio) appartiene al gruppo di emozioni chiamate "autocoscienti", che tipicamente insorgono quando violiamo, soddisfiamo o superiamo i nostri standard morali e sociali. Tuttavia, queste emozioni, e il senso di colpa in particolare, non ricadono solo su noi stessi, ma hanno anche una forte ricaduta sui comportamenti esterni e sulle relazioni interpersonali. Nel contesto di questa ricerca i risultati indicano come il senso di colpa indotto dall'avvertimento possa quindi aiutare le persone a sentirsi più empatiche nei confronti delle altre persone e della natura, il che provoca in loro la necessità di un adeguamento comportamentale pro-ambientale.

\section{'WYSS, Annika M., et al. Reactions to warnings in the climate com- mons. Journal of environmental psychology, 2021, 78: 101689}

\section{La considerazione sulle conseguenze future e la distanza temporale contribuiscono al divario ideologico nello scettici- smo sul cambiamento climatico}

Sono ancora poco esplorati i fattori che contribuiscono al divario ideologico nelle convinzioni sul cambiamento climatico (ad esempio lo scetticismo dei conservatori circa il cambiamento e la sua severità). Gli autori ipotizzano che vi siano delle differenze nelle considerazioni delle conseguenze future e nella percezione del cambiamento climatico nel tempo, tra conservatori e liberali che in parte contribuiscono a questo divario. In 3 studi svolti con 654 partecipanti di Paesi Bassi e di UK gli autori hanno dimostrato comparando conservatori ai liberali, che i primi tendono a considerare meno le conseguenze future dei loro comportamenti e percepiscono gli effetti del cambiamento climatico come più lontani in termini temporali. Inoltre hanno osservato che la distanza temporale ed in misura minore la considerazione delle conseguenze future possono in parte spiegare i livelli più alti di scetticismo sul cambiamento del clima nel lato conservatore dello spettro ideologico. Oltre a contribuire meglio a comprendere il pregiudizio di questa ideologia, i risultati hanno implicazioni sulla comunicazione del cambiamento climatico. Infatti l'ideologia politica è una caratteristica relativamente stabile e perciò resistente al cambiamento, è quindi fondamentale trovare strategie che possano avvicinare alle problematiche del cambiamento climatico i conservatori. La ricerca degli autori individua due possibile strade. In prima istanza trovare modi per incrementare il pensiero del futuro (esempio scrivendo lettere alle generazioni future) potrebbe rendere meno scettici. Inoltre focalizzarsi sul presente anziché sulle conseguenze future potrebbe aiutare a ridurre il divario ideologico. In altre parole enfatizzare gli impatti presenti (quali incendi boschivi, condizioni meteo estreme) ed evitare di menzionare gli scenari futuri ha il potenziale di colmare il divario ideologico nelle convinzioni sul cambiamento del clima.

\footnotetext{
${ }^{\circ}$ VEĆKALOV, Bojana, et al. A matter of time... consideration of future consequences and temporal distance contribute to the ideology gap in climate change scepticism. Journal of Environmental Psychology, 2021, 78: 101703
} 
6. Vedere o essere: il contatto con la natura durante il lockdown dovuto alla pandemia da Covid-19

Questo studio ha indagato come il contatto con la natura abbia giovato al benessere durante il periodo di lockdown. È stato condotto in Israele durante l'ultima settimana della prima chiusura delle attività dovuta al COVID-19, quando le persone erano costrette a rimanere in un raggio di 100 metri dalla propria abitazione. A 776 persone sono stati distribuiti questionari che includevano variabili demografiche (genere, etnia, gruppo culturale, età, perdita di reddito dovuta alla pandemia) e variabili legate al contatto con la natura (ambienti naturali vicini all'abitazione, o con possibilità di visione dalle finestre dellabitazione, o essere stati in ambienti di natura il giorno precedente) e misure legate al benessere (sentirsi positivi, negativi, vitali, felici o stressati). Prima di completare il questionario sul benessere, i partecipanti hanno preso parte ad un esperimento che ha testato l'effetto della visualizzazione di immagini della natura o di ambienti urbani sulle misure del benessere. I risultati hanno evidenziato che la natura vicina a casa o vista dalle finestre della propria abitazione contribuiva a più alti livelli di benessere e che essere stati in ambienti naturali il giorno precedente era associato con più alti livelli di sentimenti positivi. Questi benefici emergevano anche tra quelli che erano stati danneggiati economicamente dalla pandemia. La visualizzazione di immagini di natura era anch'essa associata con livelli di benessere, riducendo principalmente il livello di stress ed il sentimento negativo. I risultati che gli autori hanno ottenuto estendono così la comprensione dei benefici dell'accesso alla natura durante i periodi di stress, in modo particolare durante le emergenze quando l'accesso alla natura può essere impossibile. Inoltre le osservazioni sottolineano l'effetto duale del contatto sul benessere (aumento del sentimento positivo con riduzione del negativo e dello stress) ed indicano che l'esposizione alla natura è molto più preziosa per le donne che per gli uomini. Questi risultati sono molto importanti in considerazione delle differenze di genere dell'impatto pandemico sul benessere delle persone.

${ }^{\circ}$ MINTZ, Keren Kaplan, et al. See or Be? Contact with nature and wellbeing during COVID-19 lockdown. Journal of environmental psychology, 2021, 78: 101714

\section{Miscellanea}

\section{I I sogni della Terra: reinventare ARPA-H per la salute di persone, luoghi e pianeta}

I concetti emergenti di salute planetaria hanno aumentato la consapevolezza su come la salute degli individui, delle comunità e dei sistemi naturali della Terra siano interdipendenti. La pandemia cronica di malattie non trasmissibili (NCD) e l'aumento dell'angoscia e della disperazione sono inestricabilmente legate al degrado e alla distruzione degli ambienti e dei sottostanti sistemi di valori sociali, economici e politici. In questo articolo si esplora l'imminente istituzione dell'Agenzia per i progetti di ricerca avanzata per la salute (ARPA-H, Advanced Research Projects Agency for Health). La missione di ARPA-H è quella di "accelerare" l'innovazione, l'applicazione e l'implementazione di "scoperte sanitarie". Gli autori sostengono che la salute debba essere intesa come capacità di una persona di raggiungere il suo pieno potenziale, fisico e mentale. Nello specifico gli autori sostengono che focalizzarsi sulla malattia (a valle del sistema salute) alla fine fallirà, se non considerato in tandem con l'esperienza vissuta "a monte", che determina le vulnerabilità degli individui e delle comunità nel tempo. È necessario un approccio integrato "exposome" alla salute. "Exposome" è la scienza che studia le esposizioni ambientali totali accumulate (sia dannose che benefiche) dall'organismo nel tempo e le sue risposte biologiche. La ricerca sull'esposoma è stata resa possibile dalla nuova era delle tecnologie omiche, cioè la capacità di misurare simultaneamente un gran numero di biomolecole (proteine funzionali, metaboliti, espressione genica e influenze genetiche sul metabolismo dei farmaci/nutrienti). Questi marcatori possono aiutare a comprendere le implicazioni biologiche dell'esperienza complessiva vissuta da individui e da intere popolazioni. In particolare la scienza del microbioma ha dimostrato che i promotori della disbiosi (inquinanti ambientali, affollamento, stress acustico, stress termico, interruzioni circadiane) sono oneri sostenuti da individui e comunità socio-economicamente svantaggiati, e che lo svantaggio individuale e comunitario "entra sotto la pelle" e nell'interfaccia mente-corpo. La scienza dell'esposoma sottolinea che alcune finestre di vulnerabilità (per il rischio di malattia) e opportunità (per la promozione della salute) sono particolarmente importanti. Nel contesto di ARPA-H, ciò significa che il vantaggio o lo svantaggio socioeconomico può produrre risposte biologiche diverse a specifiche esposizioni "benefiche" o "dannose". Gli autori incoraggiano ARPA-H ad unattenta selezione degli obiettivi sulla base della conoscenza dei determinati socio economici di salute legati alle differenze di status sociale e di appartenenza etnica, oltre che alle influenze del marketing. Le agenzie come ARPA-H sono dotate di strumenti capaci di costruire una visione più ampia in grado non solo di migliorare la ricerca sull'equità sanitaria attraverso la medicina personalizzata e di precisione, ma in grado di favorire partnership intersettoriali, poiché la salute umana dipende in ultima analisi dall'affrontare le nostre numerose sfide ambientali. Sebbene ARPA-H sia un'agenzia americana svolgerà di certo un ruolo importante anche per i ricercatori internazionali, gli scienziati e gli studiosi, in particolare quelli che si occupano di ambiente e salute pubblica.

- LOGAN, Alan C.; BERMAN, Brian M.; PRESCOTT, Susan L. Earth Dreams: Reimagining ARPA for Health of People, Places, and Planet. International journal of environmental research and public health, 2021, 18.23: 12788 